\title{
Laparoscopic cholecystostomy tube placement
}

\begin{abstract}
Laparoscopic cholecystectomy is one of the most common procedures performed in the world today Acute calculus cholecystitis is the most frequent complication of cholelithiasis. Laparoscopic cholecystectomy is the best treatment for acute calculus cholecystitis when performed within 72 hours. Acute cholecystitis tends to be one of the highest risks for conversion to open surgery-due to unclear anatomy, excessive bleeding or technical complications.

Here we present 2 cases with severe acute cholecystitis that required placement of laparoscopic cholecystostomy (LC) tube. Patient subsequently underwent interval cholecystectomy, when the inflammation had subsided.
\end{abstract}

LC tube placement can be a safe alternative in such situations to avoid complications and conversion to open procedure.

Keywords: laparoscopic cholecystostomy tube, acute cholecystitis
Volume 10 Issue 3 - 2020

\author{
Abhiman cheeyandira \\ Department of General Surgery, Nazareth Hospital, USA \\ Correspondence: Abhiman Cheeyandira, Department of \\ General Surgery, Nazareth Hospital, USA, Tel 2153318897, \\ Email abhiman.cheeyndira@mercyhealth.org
}

Received: May 27, 2020 | Published: June 08, 2020

\section{Introduction}

Laparoscopic cholecystectomy is one of the most common procedures performed in the world today. Acute cholecystitis and recurrent biliary colic are the most common indications for performing laparoscopic cholecystectomy. Acute calculus cholecystitis is the most frequent complication of cholelithiasis presents one third of all surgical emergency hospital admissions. Laparoscopic cholecystectomy is the best treatment for acute calculus cholecystitis and the procedure should ideally be performed within 72 hours. Early surgery is associated with better results in comparison to delayed surgery. ${ }^{1}$

Acute cholecystitis tends to be one of the highest risks for conversion to open surgery-due to unclear anatomy, excessive bleeding or technical complications. ${ }^{2,3}$

LC tube placement remains an alternative to open surgery in cases where the gallbladder is too inflamed to allow for laparoscopic removal, and in cases where the patient is too sick to tolerate a more extensive procedure. It also provides access for diagnostic cholangiography. ${ }^{4}$

Percutaneous placement of cholecystostomy drain has been used in critically ill patients suffering from sepsis from acute cholecystitis, and patients with significant comorbidities who would not tolerate a prolonged procedure. LC tube placement can also be used where interventional radiology (IR) services are not available.

Here we present 2 cases where LC tube placement was performed in severe cholecystitis, and a subsequent interval laparoscopic cholecystectomy was performed.

\section{Case I}

Patient is a 74-year-old male from a nursing home with a past medical history of atrial fibrillation - on Coumadin, stroke, diabetes mellitus, hypertension, chronic kidney disease, and a prior history of
PEG tube -was admitted to the hospital with septic shock and diabetic ketoacidosis. He was febrile, had a white count of 19,000. He was initially admitted to the ICU and placed on intravenous inotropic support. Ultrasound scan of the liver showed thickening of the gallbladder with gallbladder stones. Patient subsequently underwent HIDA scan which was positive for cystic duct obstruction. Diagnosis of acute cholecystitis was made. As the patient was septic and high risk for cardiac complications, he underwent placement of percutaneous cholecystostomy tube by IR.

Patient was readmitted about 6 weeks later with acute changes in mental status, secondary to acute aspiration pneumonia. He developed bilateral pleural effusions and had a right-sided thoracocentesis performed. Around this time, his IR cholecystostomy drain fell out, and his liver function tests started to trend up - suggestive of ongoing acute on chronic cholecystitis. He underwent an ultrasound scan at this time which failed to visualize the gallbladder due to extensive distention of bowel gas shadows. Hence IR could not reposition the percutaneous drain. He was therefore taken to the operating room for planned laparoscopic cholecystectomy, after his acute medical condition was stabilized. Intraoperatively, there were extensive dense adhesions around the gallbladder. The gallbladder itself appeared thickened, contracted and was very friable (Figure 1). Attempt to dissect the cystic duct and cystic artery was deemed unsafe due to the significant adhesions and poor tissue friability. Hence decided to perform LCtube placement. A 12 French Foley catheter was inserted through one of the $5 \mathrm{~mm}$ port sites and placed into the fundus of the gallbladder. The balloon was then inflated within the gallbladder to secure it in place (Figure 2). A Jackson Pratt (JP) drain was inserted adjacent to it in the gallbladder fossa.

About 6 weeks later, the patient underwent laparoscopic cholecystectomy, with removal of cholecystostomy tube- uneventfully. Intraoperatively the inflammation had subsided and the previously placed cholecystostomy tube was removed. 


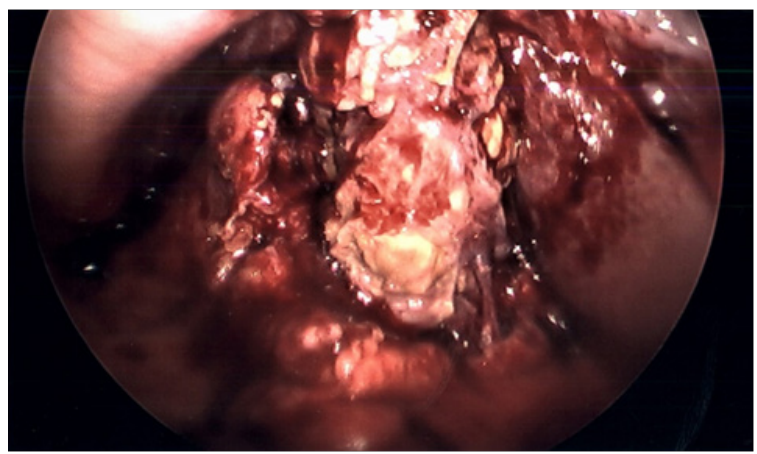

Figure I Severe acute cholecystitis.

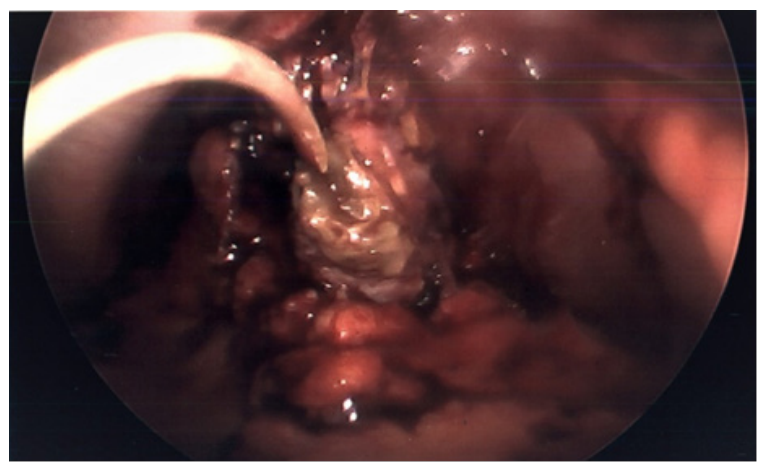

Figure 2 Laparoscopic cholecystostomy tube.

\section{Case 2}

Patient is a 49-year-old female with a history of GERD, C-section and hysterectomy-presenting with right upper quadrant pain for 2 days. White count of 20,000. Ultrasound showed thickening of gallbladder with sludge, without evidence of stones. Patient was taken for attempted laparoscopic cholecystectomy. Intraoperatively there was evidence of acute gangrenous cholecystitis with a lot of dense thick adhesions around the gallbladder. Gallbladder wall was very thick-walled and there was generalized excessive bleeding from around the liver bed. It was therefore difficult to dissect the anatomical structures. At this point it was decided to perform LC tube placement in order to avoid injury to the vital structures. A 12 French Foley catheter was inserted through a right upper quadrant $5 \mathrm{~mm}$ port site and inserted into the fundus of the gallbladder. The balloon was inflated within the gallbladder to secure it in place. A JP drain was inserted adjacent to it in the gallbladder fossa. Patient recovered quite well and was discharged home on postop day 2 after the JP drain was removed. 2 weeks later a cholecystostomy tube check was performed showed persistent cystic duct obstruction. 6 weeks from the time of the original surgery, the patient underwent elective outpatient procedure - laparoscopic cholecystectomy was performed and removal of cholecystostomy tube. Patient was discharged home the same day.

\section{Discussion}

The advent of laparoscopic cholecystectomy began in the early 1990 s, where initially there was a higher risk of conversion to open procedures due to some of the challenges encountered during surgerybetween 5 and $10 \%$ rate of conversion. Over the last 3 decades, the laparoscopic skill of the surgeons has been much more widely adopted and the conversion rate is much lower. Laparoscopic cholecystectomy can be quite challenging in especially in certain situations. Following are some of the risk factors associated with conversion to open surgery: acute cholecystitis, male patients, morbid obesity, extensive upper adhesions due to prior surgeries or trauma. Acute cholecystitis tends to be one of the highest risks for conversion to open surgery-due to unclear anatomy, excessive bleeding or technical complications. ${ }^{2,3}$

The use of percutaneous cholecystostomy tube placement by IR has been well accepted as a temporizing measure in patients with acute cholecystitis who are too unstable to undergo laparoscopic cholecystectomy. Just as catheter drainage of acute infection with interval appendectomy is accepted in patients with periappendiceal abscess, tube cholecystostomy with interval laparoscopic cholecystectomy has a role in the management of select patients with acute cholecystitis. ${ }^{6}$ These patients can be then sent home on a course of antibiotics to help them recover from the acute illness. Roughly 2 weeks later, a cholangiogram can be performed by introducing contrast through the cholecystostomy tube. If the cystic duct is found to be patent, then the cholecystostomy tube can be clamped safely. If the cystic duct is not patent, the cholecystostomy tube continues to be connected to a drainage bag. Roughly 4 to 6 weeks later when the inflammation has fully subsided, the patients can be taken electively and can undergo laparoscopic cholecystectomy. ${ }^{7}$

There may also be situations where IR may not be available such as in a rural setting, or where they are unable to perform percutaneous cholecystostomy drain placements. LC tube placement can be used as an alternative to open cholecystectomy in technically difficult cases and alternative to IR percutaneous cholecystostomy in rural hospitals without interventional radiology services. ${ }^{5}$ There are other indications for LC tube placement -such as in children with complicated choledochal cyst- where LC tube placement followed by laparoscopic cyst excision is a useful and safe procedure for the treatment of complicated choledochal cyst. This technique is also favorable from a cosmetic viewpoint because the resultant wound can be reused as the trocar insertion site at the time of laparoscopic cyst excision. ${ }^{8}$

\section{Conclusion}

Laparoscopic cholecystostomy tube placement can be a useful adjunct in situations where there is severe acute inflammation of the gallbladder and can avoid conversion to open procedure. This allows for performing interval laparoscopic cholecystectomy in a safe manner.

\section{Conflicts of interest}

The authors have no conflicts of interest to declare.

\section{Funding}

None.

\section{Acknowledgments}

None.

\section{References}

1. Acute calculus cholecystitis: Review of current best practices. World Journal of gastrointestinal surgery. 2017;9 (5):118-126.

2. Frazee RC, Roberts JW, Symmonds R, et al. What are the contraindications for laparoscopic cholecystectomy? American Journal of surgery. 1992;164(5):491-495 
3. Gadacz TR, Crist DW. Anticipating difficult cholecystectomy. Journal of laparoscopic surgery. 1992;1:69-78.

4. Kevin M. Bradley and Daniel T. Dempsey. Laparoscopic Tube Cholecystostomy: Still Useful in the Management of Complicated Acute Cholecystitis. J Laparoendosc Adv Surg Tech A. 12(3):187191.

5. Han SP. Laparoscopic cholecystostomy as an alternative to open cholecystectomy and percutaneous cholecystostomy in a rural setting. HPB. 2016;18(1):E490.
6. Eren Berber, Kristen L Engle, Andreas String, et.al. Selective use of tube cholecystostomy with interval laparoscopic cholecystectomy and acute cholecystitis. Arch surg. 2000;135(3):341-346.

7. Laparoscopic tube cholecystostomy. Surgical endoscopy. 1992;(6):285288.

8. Masaya Yamoto, Naoto Urushihara, Koji Fukumoto, et al. Usefulness of Laparoscopic Cholecystostomy in Children With Complicated Choledochal Cyst. Asian J Endosc Surg. 2015;8(2):153-157. 